\title{
5 Verfahren der Datenauswertung: Kategorienraster
}

Zusammenfassung: Im Kapitel werden sämtliche Annotationskategorien für die über 520 SN-Token dargelegt. Sie bilden die Analysegrundlage für daran anschließende inventarbezogene Untersuchungen. Die Diskussion der anzuwendenden Kategorien zeigt, dass für die Analyse des kolonialen Namenprojekts in der Metropole einige der in der vergleichenden Kolonialtoponomastik entwickelten Annotationen übernommen werden können. Darüber hinaus macht die Analyse kolonialer Mikrotoponyme in der Metropole aber weitere und andere Annotationen erforderlich. Das entwickelte Kategorienraster wird beispielhaft anhand der Annotationen für Namen der Ortspunkte Dresden und Breslau [Wrocław] präsentiert. Dabei wird deutlich, dass verlässliche strukturelle und diskursfunktionale Befunde erst durch die Annotation und Untersuchung des erstellten Gesamtinventars möglich werden.

In der programmatischen Einleitung des ersten kolonialtoponomastischen Kompendiums werden die beiden ineinander verschränkten system- und diskurslinguistischen Analyseverfahren vorgestellt, die spezifisch für die inventarbezogene Analyse kolonialer Makrotoponyme in den kolonisierten Gebieten angelegt wurden:

Einerseits leistet Diskurslinguistik eine funktionale Einordnung des einzelnen Kolonialtoponyms über das Konzept des Place-Making und die damit verbundenen Diskursfunktionen und andererseits eine Interpretation der musterhaften Vorkommen von Toponymen über die Erfassung von territorialen Strukturen. Die systemlinguistisch exakte Analyse ist dabei ebenso notwendige Voraussetzung wie die diskurslinguistische Analyse die Leistung der Linguistik zum Verständnis von Kolonialismus überhaupt erst erkennbar macht. (Stolz und Warnke 2018b: 55-56)

Auch koloniale Mikrotoponyme in den Kolonien des Kaiserreichs und im Raum der deutschen Metropole sind unter system- und diskurslinguistischen Perspektiven zu untersuchen. Die Annotationskategorien der vorliegenden Studie orientieren sich an den am Ende von Kap. 1 dargelegten zentralen Teilfragen, die toponomastische und koloniallinguistische Fragestellungen umfassen. Um „,...] den [...] Sprachgebrauch als Ausdruck kolonialer Selbstverständlichkeiten zu verstehen und damit eine Diskursanalyse des Kolonialismus als Archäologie von historischen Gewissheiten zu verstehen [...]“ (Stolz und Warnke 2018b: 59), wurden alle Analysekategorien aus der Perspektive ex ante angelegt. Die über 520 SN-Token werden ausschließlich vor dem Hintergrund ihrer historischen Verfügungsprozesse kontextualisiert, um daran anschließend konstitutive ๖ Open Access. () 2021 Verena Ebert, publiziert von De Gruyter. (c) By-ND Dieses Werk ist lizenziert unter der Creative Commons Attribution-NoDerivatives 4.0 International Lizenz. https://doi.org/10.1515/9783110718133-006 
Sprachgebrauchsmuster kolonialer Raumaneignung bzw. -besetzung in der deutschen Metropole zu untersuchen. Alle Annotationskategorien sind inventarbezogen angelegt. In der Praxis wurde jedes einzelne der über 520 kolonial motivierten SN-Token nach diversen Kriterien annotiert, um in einem nächsten Schritt ortsübergreifende Analyseabfragen hinsichtlich der damit versprachlichten kolonisatorischen Gewissheiten zu ermöglichen. Der Kriterienkatalog zeigt die einzelnen Annotationen auf, die anschließend in Unterkapiteln näher kommentiert werden. Abschließend werden sie am Beispiel der für Dresden und Breslau [Wrocław] erhobenen 21 SN-Token (Kap. 3.4) exemplarisch dargelegt.

\section{Raumzeitliche Annotationen:}

Annotation jedes SN-Tokens bzgl. des Ortstyps (+ Angabe des Ortspunktes)

\begin{tabular}{|c|c|c|}
\hline + Großstadt (G) & + Mittelstadt (M) & + annektierte Groß- und Mittelstädte \\
\hline \multicolumn{3}{|c|}{ Annotation jedes SN-Tokens bzgl. des gesellschaftspolitischen Benennungszeitraums } \\
\hline \multicolumn{2}{|c|}{$\begin{array}{l}\text { + faktische Kolonialzeit (1884-1919) bis } \\
\text { Versailler Vertrag }\end{array}$} & $\begin{array}{l}\text { + nach Versailler Vertrag } \\
\text { + Weimarer Republik bzw. Zwischen- } \\
\text { kriegszeit (1919-1933) } \\
\text { + NS-Zeit (1933-1945) }\end{array}$ \\
\hline
\end{tabular}

\section{Konstruktionsbezogene Annotationen:}

Annotation jedes SN-Tokens bzgl. des Klassifikators (KLASS)

Annotation jedes SN-Tokens bzgl. des Modifikators (MOD) (+ Angabe MOD-Token, Angabe MOD-Type) Annotation jedes SN-Tokens bzgl. der Klassenzugehörigkeit der Modifikator-Konstruktionen

\begin{tabular}{|c|c|}
\hline 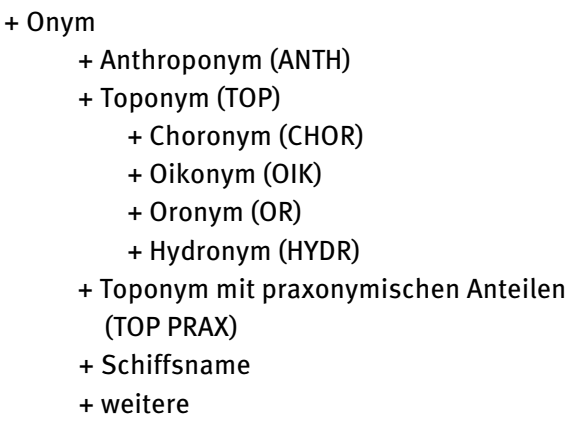 & + Appellativum (APP) \\
\hline
\end{tabular}

Annotation detoponymischer Konstruktionen bzgl. georeferenzierender Bezüge toponymischer Modifikator-Konstruktionen

\begin{tabular}{l|l}
\hline $\begin{array}{l}\text { +dt. Kolonialbesitz } \\
+ \text { Afrika } \\
\text { + Deutsch-Südwestafrika } \\
\text { + Kamerun }\end{array}$ & $\begin{array}{l}\text { + Kolonialbesitz anderer europ. Kolonial- } \\
\text { mächte }\end{array}$ \\
\hline
\end{tabular}




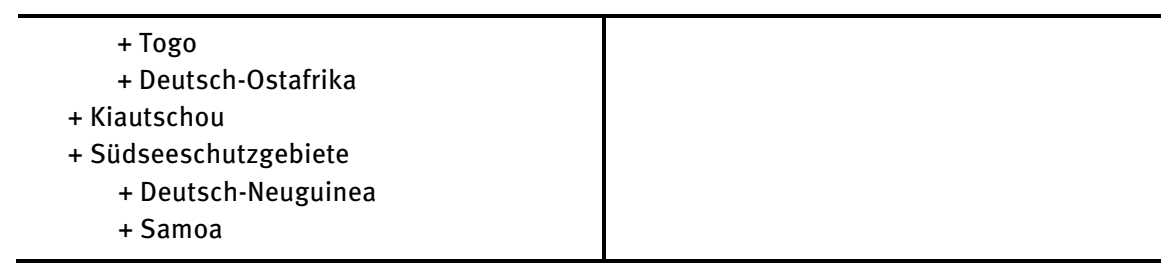

\section{Raumlinguistische Annotationen:}

Annotation jedes SN-Tokens bzgl. raumbezogener Formen kolonialer Raumreferenzierung und -belegung

\begin{tabular}{c|l}
\hline +Cluster & + Einzelbenennung \\
& + Kleincluster (+ nähere Verortung) \\
+ Großcluster (+ nähere Verortung) & \\
\hline
\end{tabular}

\section{Onomastisch-benennungskategoriale Annotationen:}

Annotation jedes SN-Tokens bzgl. Benennungsstatus

\begin{tabular}{l|l}
\hline+ Neubenennung (NEU) & + Umbenennung (UMB) \\
\hline
\end{tabular}

\section{Argumentatorisch-diskursbezogene Annotationen:}

Annotation jedes SN-Tokens bzgl. expliziter Aussagenzusammenhänge

\subsection{Raumzeitliche Annotationen}

Die beiden ersten Annotationskategorien sind weitestgehend selbsterklärend. Dabei wird den erhobenen linguistischen Sprachdaten der Ort ihres Auftretens zugewiesen. Die räumliche Zuordnung bleibt nicht auf den jeweiligen Ort beschränkt, sondern umfasst (durch entsprechende Annotation) weitere ortstypenspezifische Informationen, die die betreffenden Städte nach ihrer historischen Größe innerhalb des Untersuchungszeitraums einteilen oder auf die erst Ende der 30er Jahre erfolgte Annexion ins nationalsozialistische Deutsche Reich hinweisen. Daneben werden den Namen Informationen hinsichtlich ihrer Verfügungszeiträume zugeordnet, also danach, ob sie noch während der de factoKolonialherrschaft oder erst nach Abschluss des Versailler Vertrags in den 1920er und 1930er Jahren verfügt wurden. Ist dies der Fall, werden weitere Annotationen hinsichtlich der gesellschaftspolitischen Zeiträume (Weimarer Republik bzw. Zwischenkriegszeit oder NS-Herrschaft), in die die jeweiligen Benennungen einzuordnen sind, vorgenommen. 


\subsection{Konstruktionsbezogene Annotationen}

Die Konstruktionen des erstellten kolonialen Nameninventars zeichnen sich durch eine binäre rechtsköpfige Struktur aus und unterscheiden sich nicht von zeitgleich verfügten, nicht-kolonialen kommemorativen Straßennamen. Hinsichtlich strukturell-morphologischer Eigenschaften sind sie mit Mustern aus zeitgleich eingeschriebenen sekundären Straßennamen erklärbar (vgl. Ebert 2018: 104). Lediglich das etwas häufigere Auftreten von Konstruktionen mit anderssprachigen Erstgliedern ist in diesem Zusammenhang herauszustellen; dabei handelt es sich um Bildungen mit anderssprachigen toponymischen Erstgliedern, die, wenn auch in partieller graphematischer sowie phonetischer Integration ${ }^{101}$, auf Orte in den Kolonien referieren. Winzer (1907/10: 2) berichtet in der Zeitschrift des Allgemeinen Deutschen Sprachvereins aufgrund der „Orthographie des maliziösen Wortes Guinea“ über folgende Probleme beim Berliner Postamt:

Die Guineastraße ist [...] zu einem wahren Fluch für eine Behörde, die Post, geworden. [...] Aus einer Sammlung von Postsendungen ist zu erleben, daß das Wort Guinea in bisher nicht weniger als - dreißig Variationen vorkommt. [...] Jedenfalls gibt es auf dem Postamt N 65 infolge dieser Variationen ab und zu kleine Kolonialskandale eigener Art.

Um Anschlussfähigkeit an die vergleichende Kolonialtoponomastik herzustellen, werden die zweigliedrigen Konstruktionen kolonialer Mikrotoponyme anhand der vorgelegten Terminologie von Stolz und Warnke (2018b: 22) abstrahiert dargestellt. Der strukturelle Prototyp lässt sich auf über 520 Kolonialismen ausweiten und wird am Beispiel der Benennung Guineastraße dargelegt:

Struktureller Prototyp:

$\left[\{\text { Guinea }\}_{\text {MOD }}\{\text { straße }\}_{\text {KLAss }]_{\text {Tор }}}\right.$

Die Rolle des Kopfes kommt dem rechtsstehenden Klassifikator (abgekürzt als кLAsS) zu, der das Geo-Objekt ontologisch zuordnet. Die zweite Leerstelle wird durch das linksstehende, nominale Erstglied des Modifikators (abgekürzt als мор) besetzt. Die Kolonialismen stellen

Eigennamen hybriden Typus [dar], der appellative Strukturen mitführt. [...] Es handelt sich dabei [...] um charakterisierendes appellatives Material, das seine Semantik entfaltet, das heißt, das denotierte Objekt mitbezeichnet [...] Somit enthalten solche Komposita so-

101 Man vgl. dazu Schnee (1920 III: 714): „Windhoek [Fettdruck im Original], der holländische Name des hottentottischen Eikhams. Das Wort bedeutet "Windecke”. Seit der stärkeren Besiedlung des Landes hat man die holländische durch die der Aussprache entsprechende Schreibweise "Windhuk" [...] ersetzt". 
wohl identifizierende (onymische) als auch charakterisierende (appellative) Bestandteile [...]. (Nübling 2018: 11)

Harweg $(1983,1997)$ definiert jene Konstruktionstypen als sogenannte „Gattungseigennamen": Aufgrund ihrer Monoreferenz stellen sie Eigennamen dar, während sich der appellativische Bestandteil dieses hybriden Typus „unbeschadet des Fortbestandes seiner Gattungsnamenfunktion, dem Gesamtausdruck, von dem er ein Teil ist, funktional unterordnet“ (Harweg 1983: 160). Nübling (2018) zeigt Kriterien zur Abgrenzung appellativischer Komposita, Gattungseigennamen und reinen Namen auf. Insbesondere aufgrund ihres grammatischen Status sind die in dieser Arbeit zu untersuchenden Kolonialismen wie fast alle deutschsprachigen sekundären Straßennamen ${ }^{102}$ - eindeutig zum Typus der Gattungseigennamen zu zählen.

In der sich bisher vorrangig mit makrotoponymischen Benennungspraktiken in den kolonisierten Räumen beschäftigenden vergleichenden Kolonialtoponomastik findet dagegen eine kritische Rezeption des Konzepts der Gattung und der Gattungseigennamen statt, weil die Existenz außersprachlicher Gattungsreferenten von Ortsnamen angezweifelt wird (vgl. Döschner 2018). Dass Geoobjekte über keine objektive Gattung per se verfügen, zeigen Stolz et al. (2016: 290, Fn. 25) am Beispiel eines deutschsprachigen kolonialen Makrotoponyms auf: "JohannAlbrechts-Höhe in Kamerun is the name of a settlement or station although [...] Höhe [kursiv im Original] 'height(s)' is suggestive of an oronym (or name of mountain)”. Die These des „,charakterisierende[n] appellative[n] Material[s], das [...] das denotierte Objekt mitbezeichnet“ (Nübling 2018: 11) wird damit in Frage gestellt; stattdessen ,sind gerade die Gattungseigennamen dazu prädestiniert, Gewissheiten aus dem Kulturkreis der Benennenden zu transportieren“ (Döschner 2018: 92). Die Kritik der vergleichenden Kolonialtoponomastik an Harwegs Gattungseigennamen-Konzept setzt an den bis dato kaum untersuchten KLASS an, die gleichermaßen „für eben diese onymische Kolonisierung von Raum genutzt werden“ (Stolz und Warnke 2018b: 65). Für den Bestand kolonialer Makro- und Mikrotoponyme in den kolonisierten Gebieten erscheint diese Hypothese durch plausibel. Es ist $\mathrm{zu}$ fragen, inwieweit sich jene kritischen Überlegungen auf die Analyse des Bestandes der KLASS- und MOD-Konstruktionen des in vorliegender Arbeit erstellten Inventars kolonialer Mikrotoponyme in der deutschen Metropole auswirken muss, was zu diskutieren ist.

102 Davon ausgenommen sind Straßenbenennungen in der Zeit der DDR, deren Konstruktionen sich auch durch davon abweichende Bildungsweisen auszeichnen (vgl. Nübling et al. 2015: 249). 


\subsubsection{Klassifikatoren}

Bis auf drei Ausnahmen ${ }^{103}$ handelt es sich bei dem erhobenen Bestand kolonialer Straßennamen um zweigliedrige Konstruktionen mit rechtsköpfigen Klassifikatoren. Alle diejenigen Klassifikatoren, die ortsübergreifend für drei und mehr als drei Kolonialismen festzustellen sind, werden in der folgenden Tabelle mit den dazugehörigen absoluten tokenbezogenen Zahlenwerten dargelegt:

Tab. 1: Quantitäten an KLASS-Konstruktionen.

\begin{tabular}{lllllll}
\hline KLASS-Konstruktionen & - straße & -weg & -platz & - pfad & -allee & -kai \\
SN-Token & 424 & 57 & 18 & 6 & 3 & 3 \\
\hline
\end{tabular}

Daraus ergibt sich, dass über $80 \%$ des erhobenen Bestands kolonialer Benennungspraktiken mithilfe des usuellen appellativischen Klassifikators -straße gebildet wurden. Alle weiteren zahlenmäßig eher randständigen Phänomene weichen ebenso nicht von prototypischen Klassifikatoren nicht-kolonialer Straßennamenverfügungen der Neuzeit ab. So wurden sie im „Runderlaß des Ministers des Innern“ (17.06.1933) an alle Polizeibehörden übermittelt, die entsprechende Grundsätze für die Straßenbenennung verordneten:

Aus den Straßennamen soll erkennbar sein, daß es sich um eine Wege- oder Platzbezeichnung handelt. Zur Abwechslung sollen neben dem allgemeinen Beiwort Straße möglichst auch die Bezeichnungen Damm, Allee, Weg, Gang, Ring, Pfad, Gasse, Aue, Bahn, Steig, Steg, Wall, Graben [...] verwendet werden.

In Bezug auf die rechtsköpfigen Klassifikatoren folgen auch die genuin kolonialen Benennungsprozesse entsprechenden administrativen Bestimmungen zu neuzeitlichen Straßennamenvergaben. Die aus struktureller Perspektive festzustellende Unscheinbarkeit solcher Klassifikatoren ist im Hinblick auf die Beantwortung etwaiger spezifischer, kolonisatorischer Selbstzuschreibungskonzepte im Raum des Deutschen Reichs zu verneinen. Stattdessen fügten sie sich in usuelle Muster ein und entsprechen damit denselben administrativen Festlegungen. Für die Analyse der mit kolonialen Benennungspraktiken sprachlich

103 Bei den in Essen bzw. Hamburg und Magdeburg verfügten Benennungen Tangabucht (vgl. Stadtplan Essen 1942) bzw. Waterberg (vgl. Stadtplan im Adressbuch Hamburg 1940, Adressbuch Magdeburg 1940 II: 199) handelt es sich um Simplizia. 
evozierten kolonisatorischen Wissenseinheiten in der Metropole sind die appellativischen rechtsköpfigen Elemente allein für sich ohne Erkenntniswert.

Wenn es überhaupt etwas spezifisch ,Koloniales‘ geben sollte, dann wäre das nur in einer kombinierten, die Klassifikatoren und die Modifikatoren verbindenden Analyse erkennbar, in der bestimmte Klassifikatoren nur mit bestimmten Typen an Modifikatoren verbunden werden würden. Auch dies ist jedoch nicht der Fall: Die drei usuellen Klassifikatoren -straße, -weg und -platz werden mit einem Großteil aller MOD-Types kombiniert. Umgekehrt gilt das auch: So entspricht auch die Wahl der Klassifikatoren den inventarbezogenen aufgezeigten Quantitäten (Tab. 1), wie am Beispiel einzelner MOD-Konstruktionen gezeigt werden kann:

Tab. 2: Verteilung usueller KLASS-Konstruktionen auf ausgewählte MOD-Konstruktionen.

\begin{tabular}{llll}
\hline MOD-Konstruktionen & -straße & -weg & -platz \\
\hline Peters & 59 & 6 & 3 \\
Samoa & 9 & 2 & 1 \\
Tsingtau & 5 & - & - \\
\hline
\end{tabular}

Selbst in Fällen, in denen Kolonialismen im Zuge von Tilgungsprozessen älterer Namen verfügt wurden und sich die jeweiligen Klassifikatoren der Umbenennungsprodukte von denen der älteren Namen unterscheiden, ist nicht automatisch „ein signifikantes Merkmal von kolonialer Ortsherstellung“ (Döschner 2018: 92) festzustellen. ${ }^{104}$ In Bezug auf die zu untersuchenden Praktiken kolonialer Raumaneignung bzw. -besetzung in der deutschen Metropole haben derar-

104 Man vgl. dazu bspw. die 1939 in Berlin-Wedding erfolgte Umbenennung eines Teils der Londener Straße in Petersallee, die zeitgenössisch den Kolonialakteur Carl Peters ehren und/oder würdigen sollte: Selbst wenn der Klassifikator -allee eine Aufwertung des Straßenzugs darstellen könnte, wäre eine derartige Hypothese, dass dies in einem direkten Zusammenhang mit der kolonialen Motivik des Umbenennungsprodukts stehen muss, nicht haltbar; so sind Klassifikatoren, die teilweise im Zuge von Umbenennungsprozessen älterer Straßennamen mitverändert wurden, auch für eine ganze Reihe nicht-kolonial motivierter Straßennamen in ähnlicher Zeit zu konstatieren, bspw. die 1924 in Berlin-Zehlendorf erfolgte Umbenennung der Heidestraße in Busseallee. Die Modifizierung von Klassifikatoren in -allee ist dabei auch für Straßennamen festzustellen, deren MOD unverändert weiter bestehen blieben, bspw. das 1988 in Berlin-Wilmersdorf verfügte SN-Token Kaiserallee (seit 1950 Bundesallee). Die ursprüngliche, 1874 erfolgte Benennung war Kaiserstraße. Auch das 1872 verfügte SN-Token Frankfurter Chaussee wurde 1949 getilgt und durch Frankfurter Allee ersetzt (http://kirste.userpage.fu-berlin.de/diverse/doc/umbenennungen.html, Abruf am 28/06/2019). 
tige veränderliche Klassifikatoren aber keine Aussagekraft. Für die Beantwortung der übergeordneten Fragestellung dieser Arbeit spielen sie eine nebensächliche Rolle, sodass sie zwar im Kategorienraster berücksichtigt wurden, aber keine weiteren Annotationskriterien aufweisen.

\subsubsection{Modifikatoren: MOD-Token vs. MOD-Type}

Die linksköpfigen Modifikatoren haben die entscheidende Funktion des kommemorativen Markers zur Beantwortung der mit kolonialen Straßennamen versprachlichten Gewissheiten in der Metropole: Ihre inventarbezogene Analyse lässt daher Befunde darüber zu, welche Wissensbestände über Kolonialismus und koloniale Themen mit derartigen Benennungsprozessen im öffentlichen Raum der deutschen Gesellschaft fixiert werden sollten. Das erhobene Inventar der über 520 MOD-Token wurde für jedes einzelne SN-Token zunächst quantitativ erfasst. Daran anschließend wurde die tokenbezogene Namenform jedes singulären SN-Tokens in abstrahierter Form als Type, also „als Klasse der diesen Äußerungen zugrunde liegenden abstrakten Einheiten [...]“ (Bußmann 2008: 758), zusammengetragen. Durch den Abstraktionsprozess werden relevante übergreifende Auswertungen aus den variierenden sprachlichen Formen möglich: Die Typisierung der MOD-Token ermöglicht, dass der Bestand jener kommemorativen Marker in einem nächsten Schritt im Hinblick auf die damit versprachlichten kolonisatorischen Selbstzuschreibungskonzepte untersucht werden kann. Der Arbeitsschritt der Erstellung solcher modifikatorischer Types wird anhand von einigen Beispielen erläutert:

Tab. 3: Type-Token-Relation der Modifikatoren.

\begin{tabular}{ll}
\hline MOD-Token (Auswahl) & MOD-Type \\
\hline Von-Wissmann, Hermann-von-Wissmann, Wißmann, Wissmann & Wissmann \\
Carl-Peters, Karl-Peters, Dr.-Karl-Peters, Peters & Peters \\
Otawi, Otavi & Otavi \\
Taku-Fort, Taku & Taku(-Fort) \\
Tsingtauer, Tsingtau & Tsingtau \\
Swakopmunder & Swakopmund \\
\hline
\end{tabular}

Bei den Personennamen erfolgte die Annotation der MOD-Types ohne Vornamen sowie ohne etwaige Titel und/oder Namenszusätze wie bspw. Adelsprädikate und akademische Grade (Bsp. 1-2). Jegliche Bindestrichschreibungen mehr- 
gliedriger MOD-Token blieben damit automatisch unberücksichtigt. Auch etwaige graphematische Variationen wurden bei Nachnamen von Personennamen ${ }^{105}$ sowie bei Ortsnamen (Bsp. 3) vereinheitlicht. Daneben konnten ausführlichere und verkürzte Modifikator-Konstruktionen durch entsprechende Klammern zusammengefasst werden (Bsp. 4). Die Typisierung betrifft zudem MOD-Token wie Tsingtauer und Swakopmunder (Bsp. 5-6), die Nübling et al. (2015: 89) hinsichtlich ihres grammatischen Status - in Anlehnung an Fuhrhop (2003) - als deonymische Adjektive bezeichnen. Fuhrhop (2007: 126) nennt „die von einem Orts- oder Ländernamen abgeleitete Form auf -er, die heute als attributives flexionsloses Adjektiv aufgefasst wird“ (Duden 1998: 260), Stadtadjektive:

Das Besondere an den sogenannten Stadtadjektiven ist, dass sie zwar vorangestelltes Attribut zu Substantiven sein können, aber nicht flektiert werden. [...] Die Stadtadjektive sind [...] als Gruppe unflektierbar, das zeichnet sie gerade aus. [...] Reihenbildung für die Stadtadjektive geschieht wesentlich bei Straßennamen. (Fuhrhop 2007: 126-127)

Rein formal entsprechen die MOD-Token von Konstruktionen wie Tsingtauer Straße und Swakopmunder Straße den Adjektiven innerhalb primärer Straßennamen wie bspw. Breite Straße, Lange Straße oder Kurze Straße. Dass bei solchen kommemorativen SN-Token jedoch Reanalysen stattgefunden haben und die MOD-Token nicht mehr als Adjektive analysiert werden können, kann anhand historischer Benennungsmotiviken belegt werden:

Tsingtauer Straße. Tsingtau, Hauptstadt des früheren deutschen Pachtgebietes Kiautschou, wuchs unter deutscher Verwaltung zu einer aufblühenden Handels- und Hafenstadt heran; am 7.11.1914 nach zweimonatiger Verteidigung durch die schwache Besatzung von den Japanern und Engländern erobert. (Adressbuch München 1941 IV: 648)

Swakopmunder Straße. Stadt im ehemaligen Deutsch-Südwestafrika. (Adressbuch Leipzig 1938 II: 462)

So ist bspw. Tsingtauer Straße keine Straße, die nach Tsingtau führt oder in Tsingtau zu verorten ist. Gleiches gilt für Swakopmunder Straße. Der von Fuhrhop (2003) beschriebene Prozess der Verdunkelung hat nicht stattgefun-

105 Die unterschiedlichen Verschriftungen $\langle\mathrm{C}\rangle$ (Carl) und $\langle\mathrm{K}\rangle$ (Karl) des Anlauts $/ \mathrm{k} /$ stellen ein diachrones Phänomen dar: Schnee (1920 III: 40) führt den Namen mit $<C>$ auf. Das gilt auch für alle SN-Token, die zur faktischen Kolonialzeit und während der Weimarer Republik verfügt wurden. Die seit 1933 verfügten Benennungen werden größtenteils mit $<\mathrm{K}>$ verschriftet und sind damit offenbar auf nationalsozialistische Interessen zurückzuführen. 
den. ${ }^{106}$ Die Benennungen referieren zum Zeitpunkt ihrer historischen Namenvergabe einzig und allein auf das Substantiv, also hier auf den Ort Tsingtau bzw. Swakopmund, selbst wenn man den Modifikator aus rein formal-grammatischen Kriterien als sogenanntes „Stadtadjektiv“ bezeichnen könnte; -er kann im Hinblick auf die damit intendierten Kommemorationen der administrativen Namengeber keine Erkenntnisse liefern und wurde daher bei der Zusammenstellung der jeweiligen MOD-Types getilgt.

Dass -er vielmehr mithilfe von silbenstrukturellen Aspekten und der prosodischen Struktur der vorliegenden Strukturmuster begründet werden kann, soll anhand eines Vergleichs von Konstruktionen mit Städtenamen als Erstglied erfolgen, die mit und ohne -er (Bsp. 1), nur mit -er (Bsp. 2) und ausschließlich ohne -er (Bsp. 3-5) gebildet wurden:

Tab. 4: Prosodische Strukturen.

\begin{tabular}{llll}
\hline ohne -er & $\begin{array}{l}\text { Prosodische Struktur } \\
\text { ohne }-e r\end{array}$ & mit -er & $\begin{array}{l}\text { Prosodische Struktur } \\
\text { mit -er }\end{array}$ \\
\hline Tsingtaustraße & tsing.TAU.STRA.ße & $\begin{array}{l}\text { Tsingtauer Straße } \\
\text { tsing.TAU.er.STRA.ße }\end{array}$ & $\begin{array}{l}\text { Swakopmunder Straße } \\
\text { SWA.kop.MUN.der.STRA.ße }\end{array}$ \\
Togostraße & - & - & - \\
Togoweg & TO.go.STRA.ße & - & - \\
Togoplatz & TO.go.WEG & - & - \\
Apiastraße & TO.go.PLATZ & - & - \\
Lomeweg & A.pia.STRA.ße & - & - \\
\hline
\end{tabular}

Bei Substantiven ist die Abfolge von betonter und unbetonter Silbe aufgrund phonologischer Bedingungen fixiert (vgl. Eisenberg 1991: 47), was anhand der Klassifikatoren problemlos aufgezeigt werden kann: Für die rechtsköpfigen Bestandteile ist festzustellen, dass einsilbige (-weg, -platz) und zweisilbige (-straße) Appellativa vorliegen. Die Einsilber werden betont, bei den zweisilbigen Klassifikatoren erfolgt eine Betonung der ersten Silbe, die Schwa-Silbe am Ende ist unbetont. Das bedeutet gleichzeitig, dass jedes MOD-Token bei usuellen Bildungen mit -straße, -weg und -platz auf eine betone Silbe stößt. Werden die Endsilben der MOD-Token betont, bietet sich offenbar -er, die als stammbildende

106 „Ursprünglich wurden viele Straßen nach der Richtung genannt, in die sie führen; eine Berliner Straße sollte also nach Berlin führen. Dieser Zusammenhang ist inzwischen allgemein verdunkelt, bei Neubenennungen nicht mehr intendiert“ (Fuhrhop 2003: 101, Fn. 17). 
Schwa-Endung beim Adjektiv (bspw. heiter, munter) gilt (vgl. Eisenberg 1991: 51), an, um die Namen in usuelle Akzentmuster des Deutschen einzureihen. Dies ist bspw. bei den Konstruktionen Tsingtauer Straße und Swakopmunder Straße der Fall: Die Städtenamen Tsingtau (tsing.TAU) und Swakopmund (SWA.kop.MUND) stellen Modifikatoren mit Betonung der jeweiligen Endsilben dar; durch den Einschub der nichtbetonbaren Schwa-Silbe trifft diese auf eine betonte Silbe des Modifikators: „Wieder finden wir das vertraute Muster aus betonter und unbetonter Silbe [am Ende eines flektierenden Wortes] vor“ (Eisenberg 1991: 62). Dass die Konstruktionen vielmehr aus prosodischen Aspekten zu begründen sind, wird sodann anhand der MOD-Types Togo, Apia und Lome ${ }^{107}$ (Bsp. 3-5) deutlich: Die Konstruktionen erfolgten für das Gesamtinventar ohne -er, weil die usuellen Akzentmuster bereits vorlagen; alle zweigliedrigen Modifikatoren stellen usuelle prosodische Muster aus betonter und unbetonter Silbe dar, worauf anschließend entweder einsilbige oder zweisilbige Klassifikatoren mit entsprechender Erstbetonung folgen.

\subsubsection{Klassenzugehörigkeiten der Modifikator-Konstruktionen}

Für die ortsübergreifende Untersuchung der intendierten kolonialdiskursiven Funktionen ist eine Kategorisierung der Modifikator-Konstruktionen nach Appellativa und Onymen erforderlich. In einer früheren Studie wurde bereits für ein (Teil-)inventar solcher Kolonialismen dargelegt, dass die jeweiligen linksstehenden Modifikatoren neben singulären Appellativa vorrangig Onyme darstellen und damit ,eindeutig auf einen außersprachlichen Gegenstand, ein sog. Denotat oder Referenzobjekt“ [Fettdruck im Original] (Nübling et al. 2015: 19) verweisen (vgl. Ebert 2018: 105-109). Deonymische Konstruktionen könnten hinsichtlich der relevanten Namenklassen, die in die jeweiligen Konstruktionen eingehen, weiter kategorisiert werden. Die Annotation der kommemorativen Marker stellt ein vorrangig, aber nicht ausschließlich systemlinguistisches Verfahren dar, mit dem die über 520 MOD-Token im Hinblick auf musterhafte Strukturen kategorisiert werden können: Dabei werden sowohl historische Benennungsmotiviken als auch zeitgenössische Einträge zu den jeweiligen MOD-Types der Koloniallexika berücksichtigt, mit denen entsprechende Eigennamenklassen im Zugriff ex ante abgeleitet

107 In deutschsprachigen Plänen und Karten aus der Zeit des Kaiserreichs wurde die Stadt Lome geschrieben (vgl. Hofmann 2013). Sie wird auch im entsprechenden Artikel im Koloniallexikon von Schnee (1920: 462-463) als Lome verschriftet. Es ist davon auszugehen, dass eine phonetische Integration des Toponyms stattgefunden haben muss. 
werden können. Quantitativ dominant sind Personennamen bzw. Anthroponyme und Ortsnamen bzw. Toponyme. Toponyme wurde dabei hinsichtlich folgender relevanter Ortsnamen-Typen weiter annotiert:

RaumN oder Choronyme [Fettdruck im Original] [...] sind EN für größere geographische Flächen, Gebiete oder Räume [...]. Sie umfassen Namen für naturräumliche Objekte (Erdteile, Landschaften, Regionen, Landstriche) und künstliche, politische oder administrative Einheiten (Staaten, Länder). (Nübling et al. 2015: 208)

Unter SiedlungsN oder Oikonymen [Fettdruck im Original] [...] sind im weitesten Sinne alle Namen für Objekte zu verstehen, die von Menschen besiedelt sind und eine kleinere räumliche Ausdehnung [...] aufweisen. (Nübling et al. 2015: 212)

Unter GewässerN oder Hydronym [Fettdruck im Original] (< griech. hydor 'Wasser') [kursiv im Original] versteht man den EN von einem Fluss, Bach, Kanal, See, Teich etc. (Nübling et al. 2015: 223)

BergN bzw. Oronyme[n] [Fettdruck im Original] (< griech. oros 'Berg') [kursiv im Original]. (Nübling et al. 2015: 236)

Neben Anthroponymen und Toponymen (mit den jew. Unterklassen) treten für das Gesamtinventar zudem Modifikator-Konstruktionen auf, die auf bei Kolonialeinsätzen beteiligte Schiffe der Kaiserlichen Marine verweisen, und entsprechend annotiert wurden.

\subsubsection{Georeferenzierende Bezüge toponymischer Modifikator-Konstruktionen}

Die bisher erläuterten Annotationskriterien beziehen sich vorrangig auf Strukturbeschreibungen und damit auf formale Systematisierungen der kolonialen Mikrotoponyme, die Analysen hinsichtlich musterhafter Vorkommen der Gesamtkonstruktionen und der jeweiligen Konstituenten ermöglichen. Auf Grundlage solcher quantitativen Teilergebnisse wurde eine weitere Annotationskategorie für den Teilbestand aller detoponymischen Konstruktionen hinzugezogen, die die jeweiligen SN-Token hinsichtlich georeferenzierender Bezüge einordnet. Für alle anderen Modifikatortypen sind derartige Referenzierungen hingegen, wenn überhaupt, nur sekundär abgeleitet möglich, indem in einem einzel- bzw. tokenbezogenen Zugriff historische Benennungsmotiviken hinzugezogen werden. ${ }^{108}$ Aus diesem Grund wurde daher auch nur der Bestand aller toponymi-

108 Man vgl. dazu die unterschiedlichen Benennungsmotiviken deanthroponymischer Konstruktionen mit dem MOD-Type Wissmann: „Wissmannstraße: Nach dem Afrikaforscher Hermann von Wißmann“ (Adressbuch Bochum 1924/25 IV: 184). „Wissmannstraße: Hermann von 
schen Modifikator-Konstruktionen berücksichtigt. Die Annotation ist im sprachhistorischen Zugriff ex ante angelegt und richtet sich nach der zeitgenössisch etablierten Einteilung der deutschen Kolonialgebiete bei Schnee (1920 I: 578): Neben den Kolonien in Afrika (Deutsch-Südwestafrika, Kamerun, Togo, Deutsch-Ostafrika) und dem Gebiet an der chinesischen Ostküste (Kiautschou) fasst er den ,in der Südsee gelegenen Teil des deutschen Kolonialbesitzes, bestehend aus Deutsch-Neuguinea [...] mit Einschluß des Inselgebietes der Karolinen, Marianen, Palau- und Marshallinseln und aus Samoa“ als „Südseeschutzgebiete“ zusammen. Detoponymische Konstruktionen, die - wie die in Oranienburg verfügte Benennung Transvaalstraße (Kap. 4) - auf kolonisierte Räume anderer europäischer Kolonialmächte referieren, wurden entsprechend annotiert. Mit der Annotationskategorie werden die bisherigen weitgehend systemlinguistischen Annotationen erweitert. Sie verspricht einen Erkenntnisgewinn zur Beantwortung der Frage, inwieweit die historischen Kolonialismen hinsichtlich räumlicher Bezugnahmen auf die Kolonialgebiete mit Präferenzregeln zu beschreiben sind, also welche kolonisierten Räume besonders häufig gewürdigt werden sollten und welche dagegen offenbar nur eine marginale Rolle im Selbstverständnis der deutschen Kolonisatoren spielten.

\subsection{Raumlinguistische Annotationen}

Die Analyse kolonialer Straßenbenennungsprozesse erfordert den Miteinbezug ihrer Raumreferenzierung und -belegung und betrifft Annotationen, die eine Positionierung der historischen Kolonialismen in der unmittelbaren Raumdeskription der jeweiligen Städte vornehmen: Schon an mehreren Stellen dieser Arbeit wurde dargelegt, dass neuzeitliche Straßenbenennungen überwiegend als themenkohärente Clusterbenennungen für in direkter räumlicher Nähe zueinander liegende Straßenzüge erfolgten. Jedes einzelne koloniale SN-Token wurde dahingehend untersucht und annotiert, ob damit eine koloniale Einzelbenennung im städtischen Raum des jeweiligen Ortes vorgenommen wurde oder ob dieses stattdessen mit mindestens einer weiteren themenkohärenten kolonialen Benennung für Parallelstraßen und/oder sich kreuzende Straßenzü-

Wißmann, Afrikaforscher, 1889 Reichskommissar, 1895 Gouverneur von Deutsch-Ostafrika“ (Adressbuch Düsseldorf 1934 III: 461). „Wissmannstraße: Hermann von Wißmann, Afrikaforscher, durchquert 1880 im Dienste der deutsch-afrikanischen Ges. [d. i. Gesellschaft] Afrika, wird 1888 zum Reichskommissar für Deutsch-Ostafrika ernannt und nimmt durch Hissen der deutschen Flagge am 1.1.1891 den von Sultan von Sansibar abgetretenen Außenstrich in Besitz; 1895/96 Gouverneur von Deutsch-Ostafrika“ (Adressbuch München 1940 IV: 705). 
ge als Kolonialcluster verfügt wurde. Die bis 1945 innerhalb von klein bis groß angelegten Kolonialcluster $\mathrm{zu}$ verortenden Straßennamen wurden sogleich räumlich verortet. Damit werden erstmalig ortsübergreifende Zugriffe auf Cluster ermöglicht, mit denen u.a. die innerhalb von Kolonialclustern verfügten SNToken in vergleichender Perspektive hinsichtlich struktureller Prototypen untersucht werden können.

\subsection{Onomastisch-benennungskategoriale Annotationen}

Überhaupt muss die Frage nach der historisch-synchronen Relevanz derartiger Benennungspraktiken im Kontext kolonialer Raumaneignung bzw. -besetzung in der deutschen Metropole beantwortet werden. Hierfür wird jedes SN-Token hinsichtlich seines Benennungsstatus annotiert, also danach, ob Neu- oder Umbenennungen vorliegen. So sind bspw. ortsübergreifend die Prozesse von Tilgung und Überschreibung älterer Straßennamen im Stadtkern für Benennungen von Straßenzügen nach Adolf Hitler in nationalsozialistischer Zeit zu konstatieren (vgl. Nübling et al. 2015: 247). Für den Gesamtbestand kolonial motivierter Benennungen ist demnach $\mathrm{zu}$ untersuchen, welche tokenbezogene Verteilungen festzustellen sind und in welche historischen Kontexte die jeweiligen Teilinventare an Erst- bzw. Umbenennungen einzuordnen sind.

\subsection{Argumentatorisch-diskursbezogene Annotationen}

Neben raum-zeitlichen, konstruktionsbezogenen, raumlinguistischen und onomastisch-benennungskategorialen Annotationen der Namen selbst soll auch das Auftreten der historischen Kolonialismen in expliziten Aussagezusammenhängen erfasst werden, denn „Toponyme [...] sind Teil von Sprachgebrauchsdaten im Kontext [...] die immer auch eine konnotative Semantik besitzen“ (Stolz und Warnke 2018b: 56). In der bisherigen toponomastischen Forschung blieb eine derartige diskurslinguistische Perspektivierung auf Ortsnamen überraschenderweise gänzlich unberücksichtigt. Propositionale Einbettungen kolonialer Makrotoponyme bzw. Teilstrukturen solcher wurden in Stolz und Warnke (2018b: 58-60) anhand des Toponyms Zanzibar/Sansibar aufgezeigt, das anhand des historischen Referenzkorpus DSDK kontextualisiert wurde. In dieser Arbeit werden transtextuelle Aussagenzusammenhänge erstmalig für das koloniale Namenprojekt in der deutschen Metropole aus der Perspektive ex ante analysiert; bereits in Kap. 4 wurde dargelegt, dass für den toponymischen Modifikator der in Berlin-Wedding und Oranienburg verfügten Transvaalstraße 
durchaus propositionale Einbettungen innerhalb kolonialzeitlicher Quellen festgestellt werden können. Ähnlich wie Zanzibar/Sansibar kann Transvaal innerhalb solcher transtextuellen Aussagen als „ein Emblem für koloniale Imaginationen“ (Stolz und Warnke 2018b: 60) und damit als relevanter onymischer Bestandteil kolonialdiskursiver Wissenskonzepte herausgestellt werden. Solche transtextuellen Aussagen können bspw. dahingehend analysiert werden, inwiefern sich quantitativ dominante Modifikatoren der zusammengestellten Kolonialismen auch innerhalb solcher übertextueller Wissenseinheiten als relevant erweisen. Für die vorliegende Studie sind insbesondere die Lemmata in den Koloniallexika (vgl. Kausch 1903, Schnee 1920) zu berücksichtigen, die für fast alle MOD-Types der erhobenen Konstruktionen festzustellen sind (Kap. 4) und innerhalb derer zeitgenössische Zuschreibungen vorgenommen werden.

Von besonderem Interesse sind Propositionen, die sich nicht nur auf die MOD-Types beziehen, sondern die explizit auf das kommunikative Handeln entsprechender kolonialer Benennungsprozesse durch metasprachliche Äußerungen verweisen:

Die Metapragmatik fokussiert [...] sprachliche Handlungen, die auf sprachliche Handlungen verweisen und fragt danach, wie die Kommunikationsakteure selbst kommunikatives Handeln [...] bzw. die Umstände kommunikativen Handelns reflektieren und konzeptualisieren. (Spitzmüller 2013: 264)

Oder:

Kommunikative Handlungen, deren Objekt selbst wieder kommunikative Handlungen sind [...]. (Spitzmüller 2012: 15)

Für die deskriptiv-empirische Zusammenstellung des sprachhistorischen Inventars haben sich Benennungsmotiviken als besonders hilfreich erwiesen. Die Motiviken sind nicht nur methodisches Werkzeug, mit dem der unmittelbare Bezug kommemorativer Straßennamenverfügungen auf Kolonialismus geprüft werden konnte, sondern sie stellen zugleich metasprachliche Handlungen der zeitgenössischen administrativen Namengeberseite dar, die die jeweiligen Namenverfügungen sprachlich-diskursiv begründen. Derartige transtextuelle Einbettungen konnten für einzelne Städte in Straßenbenennungsakten, in ortsübergreifender Perspektive aber insbesondere durch die Sichtung zeitgenössischer Straßenverzeichnisse in Adress- bzw. Einwohnerbüchern (Kap. 3.3.4) erhoben werden, in denen Abschriften, teilweise auch als Extrakte oder als explizitere Verweise, administrativer Benennungsmotiviken erfolgten. Für die Beantwortung der Frage, „wie man soziale Positionierung durch Sprache als diskursives Phänomen begreifen [...] [kann]“ (Spitzmüller 2013: 284), erfolgte eine Annotation derjenigen SNToken, für die solche metasprachlichen Texte vorliegen. 


\subsection{Annotationskriterien am Beispiel der für Dresden und Breslau [Wroctaw] erhobenen SN-Token: Zwischenergebnisse}

Das zu Beginn des Kapitels dargelegte Kategorienraster mit den in den jeweiligen Unterkapiteln erläuterten Annotationskriterien werden abschließend am Beispiel der in Dresden und Breslau [Wrocław] verfügten kolonial motivierten SN-Token erklärt, für die bereits in Kap. 3.4 Identifizierungs- und Inventarisierungsarbeiten erläutert wurden. Für die beiden Ortspunkte wurden 21 kolonial motivierten SN-Token erhoben:

Dresden: Godeffroystraße, Karl-Peters-Straße, Leutweinstraße, Lüderitzstraße, Nachtigalstraße, Rohlfsstraße, Swakopmunder Straße, Windhuker Straße, Wissmannstraße, Woermannstraße.

Breslau [Wrocław]: Apiastraße. Dualastraße, Heinrich-Schnee-Straße, Karl-Peters-Straße, Lettow-Vorbeck-Straße, Lüderitzstraße, Samoastraße, Tangastraße, Togostraße, Windhukstraße, Wissmannstraße.

Für jedes einzelne SN-Token wurden die dem Kategorienraster entsprechenden Annotationen vorgenommen. Ein entsprechender Auszug aus dem Annotationsraster findet sich in Appendix 3.

Im Folgenden werden Zwischenergebnisse der vorgenommenen Annotationen am Beispiel der 21 Kolonialismen erklärt. Gleichzeitig wird aufgezeigt, welche bisher ungeklärten ortsübergreifenden Fragen durch das Annotationsraster beantwortet werden können.

Die für die zwei Großstädte erhobenen 21 Kolonialismen sind in die de factoKolonialzeit (Dresden) und in die Zeit des Nationalsozialismus zu verorten (Dresden, Breslau [Wrocław]). Eine erste Auswertung der konstruktionsbezogenen Annotationen zeigt, dass die in beiden Städten verfügten Namen prototypische Strukturen aufweisen; die zweigliedrigen Konstruktionen werden allesamt mithilfe des usuellen rechtsköpfigen Klassifikators -straße gebildet. Aber auch im Hinblick auf die MOD-Types zeigen die Kolonialtoponyme bereits vier Dubletten auf:

Dubletten der in Dresden und Breslau [Wrocław] verfügten Kolonialismen
$\left[\left\{\text { Peters }_{\mathrm{ANTH}}\right\}_{\mathrm{MOD}}-\left\{\text { straße } \text { APP }_{\mathrm{KLASS}}\right]_{\mathrm{TOP}}\right.$
$\left[\left\{\text { Lüderit } \boldsymbol{z}_{\mathrm{ANTH}}\right\}_{\mathrm{MOD}}-\left\{s t r a ß \boldsymbol{e}_{\mathrm{APP}}\right\}_{\mathrm{KLASS}}\right]_{\mathrm{TOP}}$

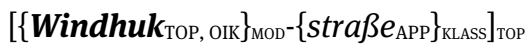
$\left[\left\{\text { Wissmann }_{\mathrm{ANTH}}\right\}_{\mathrm{MOD}}-\left\{\text { straße }_{\mathrm{APP}}\right\}_{\mathrm{KLASS}}\right]_{\mathrm{TOP}}$

Bei den Dubletten handelt es sich um drei deanthroponymische Konstruktionen und eine deoikonymische Konstruktion. Die Annotation sämtlicher 21 SN-Token ergibt, dass es sich auch bei den weiteren Modifikator-Konstruktionen um Anthro- 
ponyme und Toponyme (mit teilweise praxonymischen Anteilen) handelt. In vergleichender Perspektive ist zu konstatieren, dass die Anzahl an toponymischen Modifikatoren der für Breslau [Wrocław] erhobenen SN-Token (6) größer ausfällt als die für Dresden (2). Jene acht toponymischen MOD-Types wurden gleichzeitig hinsichtlich ihrer jeweiligen Unterklassen annotiert: Neben singulären Choronymen (Togo, Samoa) handelt es sich bei dem Großteil der in die zwei Großstädte verfügten Kolonialismen um Oikonyme (Windhuk, Apia, Swakopmund, Duala). Ausgehend von solchen toponomastischen Formen und ihrer quantitativen Erfassung sind die mit derartigen Kolonialtoponymen einhergehenden Diskursfunktionen zu untersuchen: Die für Dresden und Breslau [Wrocław] aufgezeigten strukturellen Dubletten verweisen auf deutsche Kolonialakteure (Carl Peters, Adolf Lüderitz und Herrmann von Wissmann) sowie auf eine Stadt in den kolonisierten Gebieten (Windhuk). Und auch alle weiteren für die beiden Orte erhobenen Kolonialismen stellen deanthroponymische, dechoronymische und deoikonymische Konstruktionsmuster dar, die sich auf weitere Kolonialakteure und -orte im kolonisierten Raum beziehen. Anhand konstruktionsbezogener Annotationen des erhobenen (Gesamt-)bestands der über 520 MOD-Konstruktionen kann geklärt werden, ob die für nur zwei Ortspunkte aufgezeigten prototypischen Strukturmuster den ortsübergreifenden kolonialen Benennungsprozessen in der deutschen Metropole entsprechen oder davon abweichen.

Die Annotation der Konstruktionen mit toponymischen Modifikatoren hinsichtlich georeferenzierender Bezüge zeigt im Ortsvergleich Unterschiede auf: Die zwei mit oikonymischen Modifikatoren verfügten Benennungen (Swakopmunder Straße, Windhuker Straße) in Dresden referieren auf die Kolonie DeutschSüdwestafrika. Für die sechs in Breslau [Wrocław] verfügten SN-Token mit toponymischen Modifikatoren sind dagegen unterschiedliche räumliche Bezüge festzustellen; georeferierende Bezüge auf die Kolonie Samoa erfolgt durch zwei SNToken mit entsprechendem choronymischen (Samoastraße) bzw. oikonymischen (Apiastraße) Modifikator. Die Kolonie Togo in Afrika ist durch das SN-Token mit gleichnamigem choronymischen Modifikator (Togostraße) vertreten. Eine räumliche Bezugnahme auf die Handelskolonie an der Südostküste der chinesischen Provinz findet nicht statt. Daneben referieren zwei weitere Kolonialismen auf Orte in Deutsch-Südwestafrika (Windhukstraße) und Kamerun (Dualastraße). Etwaige georeferenzierende Präferenzen kolonialer Benennungspraktiken können durch die Annotation des ortsübergreifend erhobenen Teilbestandes aller detoponymischen Konstruktionen herausgestellt werden.

Die Produkte der in den öffentlichen Raum der beiden Städte verfügten Kolonialismen erfolgten in Form von themenkohärenten Clustern, die in die städtischen Peripheriebereiche $\mathrm{zu}$ verorten sind: Räcknitz/Zschertnitz grenzt an die Dresdner Südvorstadt an; die betreffenden Straßenzüge in Breslau [Wrocław] 
liegen am „Stadtrand [...] Mochbern“ (Thum 2003: 360). Der für Dresden erhobene Clustertyp unterliegt einer zeitlichen Schichtung: Koloniale Straßenbenennungen aus der de facto-Kolonialzeit werden in nationalsozialistischer Zeit mit weiteren themenkohärenten Straßennamenverfügungen erweitert - nicht zuletzt offenbar auch deshalb, weil in unmittelbarer Nähe neue Straßenzüge errichtet wurden (Kap. 3.4.1). Trotz partiell gleicher und ähnlicher Strukturtypen liegt für Breslau [Wrocław] ein davon abweichender Clustertyp vor: Alle elf Kolonialtoponyme wurden erst nach 1933 verfügt, ein Interesse an kolonialen Benennungsprozessen ist damit erstmalig für die nationalsozialsozialistische Zeit festzustellen (Kap. 3.4.2). Durch die Annotation des (Gesamt-)inventars - unter Berücksichtigung etwaiger raumlinguistischer Muster und ihrer zeitlichen Benennungsumstände kann nachvollzogen werden, für welche(n) der drei Untersuchungszeiträume (de facto-Kolonialzeit, Weimarer Republik, NS-Zeit) ein ortsübergreifend-nationales Interesse an kolonialen Straßennamen festzustellen ist.

Bei allen elf SN-Token handelt es sich um Neu- bzw. Erstbenennungen in den städtischen Peripheriebereichen. Durch die benennungskategoriale Annotation aller über 520 SN-Token kann herausgefunden werden, welche Verteilungen sich hinsichtlich Neu- bzw. Erst- und Umbenennungen ergeben. Auf Grundlage solcher Annotationen kann die Frage nach der historisch-synchronen Relevanz kolonialer Fixierungspraktiken in der deutschen Metropole durch entsprechende Straßenvergabeprozesse beantwortet werden.

Metasprachliche Äußerungen, die die jeweiligen historischen Verfügungsprozesse von administrativer Namengeberseite explizit kommentieren, liegen für fünf der in Dresden noch in der faktischen Kolonialzeit verfügten kolonialen Straßennamen anhand von Ortsgesetzblättern vor, in denen die Namenverfügungen mit der jeweiligen Benennungsmotivik vom Rat zu Dresden mit ,allerhöchster Genehmigung“ (OGB 1913: 39) aufgelistet wurden. Die Texte stellen die deanthroponymischen Benennungen über ihre koloniale Motivik hinaus in den Fokus weiterer Interessen: So wird bspw. auch auf das wirtschaftliche Engagement der jeweiligen Personen (Großkaufmann, Reeder) hingewiesen. Die jüngeren Namenverfügungen wurden ebenfalls in entsprechenden Ortsgesetzblättern (vgl. OGB 1934, OGB $1938^{109}$ ) bekannt gegeben, allerdings ohne Angabe von Benennungsmotiviken; auch wurden keine Erläuterungen zur Fortführung themenkohärenter Straßennamen in nächster Nähe zu den älteren Kolonialismen vorgenommen. Für die allesamt in nationalsozialistischer Zeit in Breslau [Wrocław] als Cluster verfügten Kolonialismen konnten nur für drei deanthroponymische Benennungen zeitgenössische Motiviken erhoben werden; sie stam-

109 Der Beschluss im OGB von 1938 findet nicht mehr durch den Rat zu Dresden, sondern durch den „Oberbürgermeister der Landeshauptstadt Dresden“ statt. 
men aus dem Einwohnerbuch von 1943, das ,unter Benutzung amtlicher Quellen“ (Adressbuch Breslau 1943: Untertitel) vom Verlag August Scherl Nachfolger erstellt wurde. ${ }^{110}$ Es ist davon auszugehen, dass es sich dabei um Abschriften der in den jeweiligen administrativen Verordnungen aufgeführten Benennungsmotiviken handelt, die allenfalls verdichtet wurden oder explizitere Verweise anführen (Kap. 3.3.4). Sie verorten die Personen Schnee, Lettow-Vorbeck und Wissmann innerhalb ihrer kolonialen Aktivitäten in der Kaiserzeit. Die Motivik des verfügten SN-Tokens Heinrich-Schnee-Straße bezieht sich zudem explizit auf vergangene kolonisatorische Machtkonstellationen (ehemalig[er]). Wenngleich derartige metasprachlichen Texte nur für einen Teil der historischen Kolonialismen erhoben werden konnten, ist $\mathrm{zu}$ fragen, inwieweit damit ebenfalls kolonisatorische Gewissheiten versprachlicht werden sollten. Gerade für die nach 1919 verfügten kolonialen Straßennamen ist zu untersuchen, inwieweit auch innerhalb von solchen Benennungsmotiviken gängige kolonialrevisionistische Argumentationsmuster (vgl. Kämper 2016) vorzufinden sind.

Bei den zuvor erläuterten Aspekten handelt es sich nur um tendenzielle Zwischenbefunde, mit denen erste Eindrücke im Hinblick auf das koloniale Namenprojekt in der deutschen Metropole gewonnen werden können. Dass die Leitfragenkomplexe dieser Arbeit ortsübergreifend und damit inventarbezogen $\mathrm{zu}$ analysieren sind, wurde mehrfach dargelegt. Die nun folgenden Großkapitel legen die Ergebnisse vor, die aus der Datenannotation der über 520 kolonial motivierten SN-Token gewonnen werden konnten: Die Datenauswertung umfasst zuallererst quantitative Befunde $\mathrm{zu}$ zeiträumlichen und konstruktionsbezogenen Distributionen (Kap. 6). Anschließend wird der Gesamtbestand der MOD-Token hinsichtlich musterhafter sprachstruktureller und sich daraus ergebenden diskursfunktionalen Eigenschaften dargelegt (Kap. 7). Dies erfolgt unter Berücksichtigung propositionaler Einbettungen der Modifikatoren und entsprechender Namenverfügungen, aus denen sich weitere Hinweise auf kolonisatorische Zuschreibungen ableiten lassen. Daraufhin werden raumlinguistische Prototypen kolonialer Referenzierung und -belegung erläutert (Kap. 8). Zuletzt wird der Frage nachgegangen, welche inventarbezogenen Distributionen sich hinsichtlich des Benennungsstatus ergeben und in welche historischen Kontexte die Prozesse kolonialer Erst- bzw. Umbenennung eingeordnet werden können (Kap. 9).

110 Der Verlag hat in nationalsozialistischer Zeit eine ganze Reihe weiterer Adressbücher herausgegeben, die zur Erhebung kolonialer Namenvergaben in weiteren Städten des Ortskorpus genutzt wurden (vgl. Adressbuch Frankfurt am Main 1931, Adressbuch Frankfurt am Main 1941, Adressbuch Halle a. d. Saale 1943, Adressbuch Hannover 1940, Adressbuch Kiel 1940, Adressbuch Leipzig 1938, Adressbuch Magdeburg 1940, Adressbuch Stettin 1941). 